\title{
PERIPHERAL RETINAL DEGENERATION
}

Peripheral retinal degenerations are very common, and it is important to recognise their various patterns in order to judge their significance. Lattice degeneration, pigmentary anomalies and cystic degeneration will be considered here.

\section{Lattice Degeneration}

Lattice degeneration probably arises either as a chorioretinal atrophy or as a capillary sclerosis. It appears as a zig-zag pattern of small white lines in the equatorial region of the fundus. These lines may sometimes be traced to larger sclerosed or sheathed vessels. Within the lattice work cystic changes occur that may lead to the formation of multiple small round holes in the retina (Fig. 1). Histologically the affected area shows a thinned atrophic retina, blood vessels with thickened walls, and sometimes irregular pigment accumulation. A portion of this thinned retina may tear, giving rise to a large irregular flap that is sometimes only seen to open on scleral depression. (A white dot lattice degeneration with similar complications has also been described.) As lattice degeneration may give rise to multiple small retinal holes or large irregular retinal flaps predisposing to retinal detachment, prophylactic light coagulation around the lesion (and including at least a margin of normal retina) should be undertaken, particularly where there are open retinal holes, where there is a retinal detachment in the other eye, or where there is a family history of detachment.

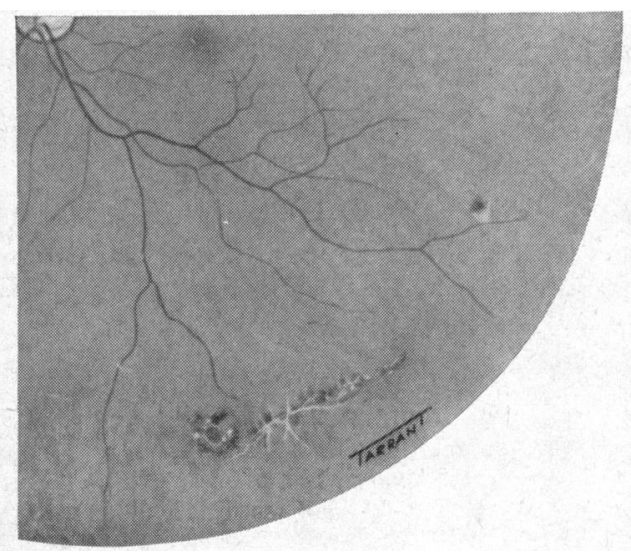

FIG. 1.-Lattice degeneration with holes and pigment.

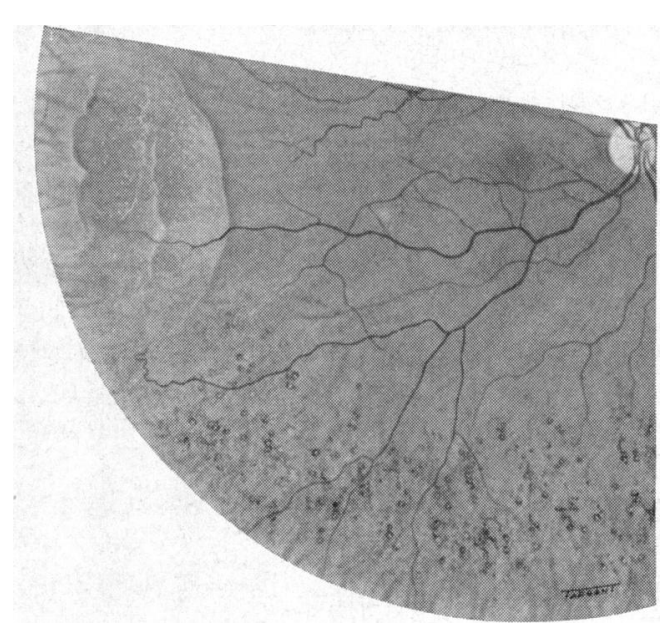

FIG. 2.-Cobblestone degeneration below. On the left is seen an area of retinoschisis with white dots.

\section{Pigmentary Anomalies}

Pigment anomalies, either with well-defined clinical patterns or as irregular accumulations, occur especially in the older age groups and in high myopes. While the classical pigmentary degeneration of the retina is rare, cobblestone and paving-stone degenerations are common. Cobblestones (Fig. 2) are very small rings of pigment commonly seen in clumps in the peripheral fundi of the elderly. These benign lesions represent defects in the retinal pigment epithelium with underlying excrescences of Bruch's membrane. Paving stones (Fig. 3) are much larger lesions resembling areas of inactive choroiditis, again common in high myopes and old people. There is however no evidence of inflammation and pathologically the lesions resemble patches of degenerative myopia. They are entirely benign although rarely tiny retinal holes may appear at the edge of one of these patches. 


\section{Peripheral Cystic Degeneration}

Peripheral microcystic degeneration of the retina (Fig. 4) is almost universal over the age of 20 years. Its clinical interest lies in its relations both to multiple hole formation often found in aphakic retinal detachment, and to retinoschisis. While the histological picture is familiar, clinically these lesions appear as small or minute pinkish-brown cysts surrounded by greyish-white retina, the whole superimposed on a background of irregular pigmentation usually situated proximal to the ora serrata. The cysts blanch with pressure, but true holes can often be identified with the magnification afforded by the three-mirror goniolens. Under normal circumstances these lesions must be considered benign.

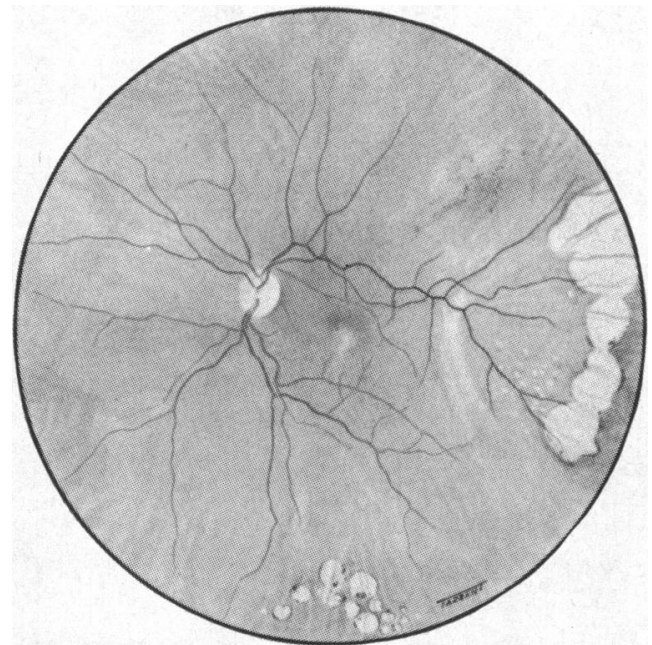

FIG. 3.-Paving-stone degeneration. On the right the shadowing of the retinal vessels indicates a shallow retinoschisis.

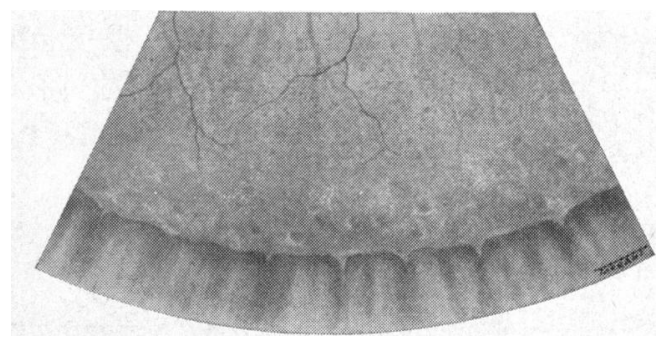

FIG. 4.-Peripheral microcystic degeneration.

Retinoschisis (Figs 2 and 3) is an uncommon condition that histologically appears to represent an abnormal extension of the splitting of the neuro-retina that occurs in microcystoid degeneration. Retinoschisis may take two forms. The first is the giant dome-shaped retinal cyst, with its diaphanous shagreen surface representing the inner leaf of the split retina, sometimes occurring symmetrically at the periphery of the two eyes. The second form, which may occur spontaneously or as part of a hereditary picture, is more difficult to diagnose. Here the inner leaf of the split retina does not bulge into the vitreous, but is often only slightly separated from its outer leaf (Fig. 3). However, the diaphanous surface is still apparent and this may be so thin as to be punctured by holes. Only if holes appear in the posterior layer can a true retinal detachment arise. Helpful diagnostic signs include the presence of tiny white dots on the surface of the retinoschisis (Fig. 2), blanching of the fundus over an area of scleral depression, and (unlike a true detachment) a white reaction to light coagulation. Naturally a split retina remains a functionless retina, and the methods available for stopping the progress of this condition are not entirely satisfactory.

Illustrations:

INSTITUTE OF OPHTHALMOLOGY.
R. K. BLACH,

MOORFIELDS EYE HOSPITAL, LONDON, E.C.1. 